\title{
The Gut-Brain Axis: Literature Overview and Psychiatric Applications
}

\author{
LT Janine Faraj, MD, USN, MC; LT Varun Takanti, MD, USN, MC; and Hamid R. Tavakoli, MD
}

Importance: Literature exploring the relationship between the intestinal microbiome and its effects on general health and well-being has grown significantly in recent years, and our knowledge of this subject continues to grow. Mounting evidence indicates that the intestinal microbiome is a potential target for therapeutic intervention in psychiatric illness and in neurodegenerative disorders such as Alzheimer disease. It is reasonable to consider modulating not just a patient's neurochemistry, behavior, or cognitive habits, but also their intestinal microbiome in an effort to improve psychiatric symptoms.
Observations: In this review paper, we show that intestinal microbiota possess the ability to directly influence both physical and mental well-being; therefore, should be included in future discussions regarding psychiatric treatment.

Conclusions: Clinicians are encouraged to consider patients' gut health when evaluating and treating psychiatric conditions, such as anxiety and depression. Optimization and diversification of gut flora through the use of psychobiotics-probiotics that confer mental health benefits-may soon become standard practice in conjunction with traditional psychiatric treatment modalities such as pharmacotherapy and psychotherapy.
Janine Faraj is a General Medical Officer at Naval Surface Forces Atlantic, Medical Readiness Division, Norfolk, Virginia. Varun Takanti is a Resident Physician in the Department of Anesthesiology at Rush University Hospital in Chicago, Illinois. Hamid Tavakoli is the head of Psychiatry Consultation-Liaison Services at the Naval Medical Center, Portsmouth, Virginia. Correspondence: Hamid Tavakoli (hamid.r.tavakoli.civ@mail.mil)

Fed Pract. 2021;38(8). Published online August 17. doi:10.12788/fp.0159
T he gut-brain axis (GBA) refers to the link between the human brain with its various cognitive and affective functions and the gastrointestinal (GI) system, which includes the enteric nervous system and the diverse microbiome inhabiting the gut lumen. The neurochemical aspects of the GBA have been studied in germ-free mice; these studies demonstrate how absence or derangement of this microbiome can cause significant alterations in levels of serotonin, brain-derived neurotrophic factor, tryptophan, and other neurocompounds. ${ }^{1,2}$ These neurotransmitter alterations have demonstrable effects on anxiety, cognition, socialization, and neuronal development in mice. ${ }^{1,2}$

Current evidence suggests that the GBA works through a combination of both fastacting neural and delayed immune-mediated mechanisms in a bidirectional manner with feedback on and from both systems. ${ }^{3}$ In addition to their direct effects on neural pathways and immune modulation, intestinal microbiota are essential in the production of a vast array of vitamins, cofactors, and nutrients required for optimal health and metabolism. ${ }^{4}$ Existing research on the GBA demonstrates the direct functional impact of the intestinal microbiome on neurologic and psychiatric health.

We will review current knowledge regarding this intriguing relationship. In doing so, we take a closer look at several specific genera and families of intestinal microbiota, review the microbiome's effects on immune function, and examine the relationship between this microbiome and mental disease, using specific examples such as generalized anxiety disorder (GAD) and major depressive disorder (MDD). We seek to consolidate existing knowledge on the intricacies of the GBA in the hope that it may promote individual health and become a standard component in the treatment of mental illness.

\section{DIRECT ACTIVATION OF NEURONAL PATHWAYS}

Vagal and spinal afferent nerve pathways convey information regarding hormonal, chemical, and mechanical stimuli from the intestines to the brain. ${ }^{3}$ These afferent neurons have been shown to be responsive to microbial signals and cytokines as well as to gut hormones. This provides the basis for research that presumes that neurobehavioral change may ensue from manipulating the gut microbes emitting these chemical signals to which these afferent neurons respond. ${ }^{3}$ Using these same pathways, efferent neurons of the parasympathetic and sympathetic nervous systems can modulate the intestinal environment by altering acid and bile secretion, mucous production, and motility. This modulation can directly impact the relative diversity of intestinal flora, and in more extreme states, may result in bacterial overgrowth. ${ }^{5}$ Of particular relevance to mental health $(\mathrm{MH})$ is that the frequency of migrating motor complexes that promote peristalsis can be directly influenced by readily modifiable behaviors such as sleep and food intake, which can 
cause one bacterial species to dominate in a higher percentage..$^{5}$ This imbalance of gut microbes has been implicated in contributing to somatic conditions, such as irritable bowel syndrome (IBS), which the literature has shown is related to psychiatric conditions such as anxiety. ${ }^{5}$

\section{The Microbiome and Host Immunity}

The GI tract is colonized with commensal microorganisms from dozens of bacterial, archaeal, fungal, and protozoal groups. ${ }^{6}$ This relationship has its most classical immunologic interaction in the toll-like receptors. These receptors are on the lymphoid Peyer patches of the GI tract, which sample microorganisms and develop immunoglobulin (IgA) antibodies to them. Evidence exists that commensal microflora play a critical role in the regulation of host inflammatory response. ${ }^{7}$

The relationship between the microbiome and the immune system remains poorly understood, yet evidence has shown that the use of probiotics may reduce inflammation and its sequelae. Probiotics have been shown to have a beneficial effect on autoimmune diseases, such as Crohn disease and ulcerative colitis, specifically with certain strains of Escherichia coli (E coli) and a proprietary probiotic from VSL pharmaceuticals. ${ }^{8,9}$ However, these interventions are not without risk. Fecal microbiota transplants have a risk of transferring unwanted organisms, potentially including COVID-19. ${ }^{10}$ Additionally, the use of probiotics is generally discouraged in immunocompromised, chronically ill, and/or hospitalized patients, as these patients may be at greater risk of developing probiotic bacteremia and sepsis. ${ }^{11}$

Studies have also demonstrated that ingesting probiotics may decrease the expression of pro-inflammatory cytokines. ${ }^{11}$ In a study comparing patients with ulcerative colitis who were prescribed both sulfasalazine and probiotic supplements vs sulfasalazine alone, patients who took the probiotic supplements were shown to have less colonic inflammation and decreased expression of cytokines such as IL- 6 , tumor necrosis factor- $\alpha(\mathrm{TNF}-\alpha)$, and nuclear factor-к $\beta .^{12}$

\section{Gut-Specific Bacterial Phyla}

Over the past decade, much attention has been paid toward 2 bacterial phyla that com- promise a large proportion of the human gut microbiome: Firmicutes and Bacteroidetes. Intestinal Firmicutes species are predominantly Gram positive and are found as both cocci and bacilli. Well-known classes within the phylum Firmicutes include Bacilli (orders Bacillales and Lactobacillales) and Clostridia. The phylum Bacteroidetes is composed of Gram-negative rods and includes the genus Bacteroides-a substantial component of mammalian gut biomes. The ratio of Firmicutes to Bacteroidetes, also known as the $\mathrm{F} / \mathrm{B}$ ratio, have shown fascinating patterns in certain psychiatric conditions. This knowledge may be applied to better identify, treat, and manage such patients.

Regarding bacterial phyla and their relationship to mood disorders, interesting patterns have been observed. In one population of patients with anorexia nervosa (AN) lower diversity within classes of Firmicutes bacteria was observed compared with age- and sexmatched controls without AN..$^{13}$ As patients were re-fed and treated in this study, there was a significant corresponding increase in microbiome diversity; however, the level of bacterial diversity in re-fed patients with AN was still far less than that of patients in the control group. In patients with AN with comorbid depression, diversity was noted to be exceptionally reduced. Similarly, patients with AN with a more severe eating disorder psychopathology demonstrated decreased microbial diversity. ${ }^{13}$

The impact of intestinal microbiome diversity and relative bacterial population density in $\mathrm{MH}$ conditions such as anxiety, depression, and eating disorders remains an intriguing avenue worth further exploring. Modulating these phenomena may reduce overall dysfunction and serve as a possible treatment modality.

\section{ANXIETY AND THE MICROBIOME}

GAD is characterized by decreased social and occupational functioning. Anxiolytic pharmacotherapy combined with psychotherapy are the current mainstays of GAD treatment. Given the interplay of the gut microbiome and $\mathrm{MH}$, probiotics may prove to be a promising alternative or adjunct treatment option.

The human stress response is enacted largely through the hypothalamus-pituitaryadrenal (HPA) axis. Anxiety and situational 
fear trigger a stress response that results in increased cortisol being released from the adrenal glands, thereby disrupting typical GI function by modifying the frequency of migrating motor complexes, the electromechanical impulses within the smooth muscle of the stomach and small bowel that allow for propagation of chyme. This, in turn, has downstream consequences on the composition of the intestinal microbiome. ${ }^{14} \mathrm{~Pa}$ tients with GAD have a lower prevalence of Faecalibacterium, Eubacterium rectale, Lachnospira, Butyricioccus, and Sutterella, all important producers of short-chain fatty acids (SCFA). ${ }^{15,16}$ Diminished SCFA production has been linked to intestinal barrier dysfunction, contributing to increases in gut endothelial permeability and facilitating a proinflammatory response with resultant neural feedback loops. ${ }^{17,18}$ Indeed, proinflammatory cytokines, namely C-reactive protein (CRP), interleukin 6 (IL-6), and TNF- $\alpha$ were found to be elevated in patients with diagnosed GAD. ${ }^{19}$ These proinflammatory cytokines are critical in neurochemical modulation as they inhibit the essential enzyme tetrahydrobiopterin, a cofactor of monoamine synthesis, thereby decreasing the monoamine neurotransmitters serotonin, dopamine, and norepinephrine. ${ }^{20}$ Decrease in the monoamine neurotransmitters serves as the lynchpin for the monoamine hypothesis of both anxiety and depression and currently guides our choice in pharmacotherapy. ${ }^{21}$

Anxiolytic pharmacotherapy targets the neurochemical consequences of GAD to ameliorate social, functional, and emotional impairment. However, the physiology of the gut-brain feedback loop in GAD is an attractive target for the creation and trialing of probiotics, which can rebalance intestinal flora, reduce inflammation, and allow for increased synthesis of monoamine neurotransmitters. Indeed, Lactobacillus and Bifidobacterium have been shown to possess anxiolytic properties by increasing serotonin and SCFAs while reducing the HPA adrenergic response. ${ }^{22}$

\section{Depression and the Microbiome}

MDD significantly diminishes quality of life and is the leading cause of disability worldwide, affecting nearly 350 million individuals. ${ }^{23}$ Psychotherapy in conjunction with pharmacotherapy aimed at increasing cerebral serotonin availability are the current mainstays of MDD treatment. Yet the brain does not exist in isolation: It has 3 known methods of bidirectional communication with the GI tract via the vagus nerve, immune mediators, and bacterial metabolites. ${ }^{24,25}$

The vagus nerve (vagus means wandering in Latin), is the longest nerve of the autonomic nervous system (ANS) and historically has been called the pneumogastric nerve for its parasympathetic innervation of the heart, lungs, and digestive tract. Current research supports that up to $80 \%$ of the fibers within the vagus nerve are afferent, relaying signals from the GI tract to the brain. ${ }^{26}$ Therefore, modulation of vagus nerve signaling may theoretically impact mental health. Indeed, studies have demonstrated clinically significant improvement in patients with treatment-resistant depression who underwent vagal nerve stimulation (VNS). ${ }^{27} \mathrm{Al}-$ though the mechanism by which it exerts its mood-modulating activity is not well understood, recent human and animal studies indicate that VNS may alter central neurotransmitter levels, having demonstrated the ability to increase serotonin levels. ${ }^{25}$ Also the vagus nerve possesses the ability to differentiate between pathogenic and nonpathogenic gut microorganisms; beneficial gut flora emit signals within the gut lumen, which in turn, are transmitted through afferent vagus nerve fibers to the brain, effecting both anti-inflammatory and mood-modulating responses. $^{25,28}$

Immunomediators involving intestinal microbiota also are known to play a critical role in the pathophysiology of MDD. Depression is closely tied to systemic inflammation; both are hypothesized to have played a role in the evolutionary response to fighting infection and healing wounds. ${ }^{29}$ With regard to the gut, MDD is associated with increased GI permeability, which allows for microorganisms to leak through the intestinal mucosa into the systemic circulation and stimulate an inflammatory response. ${ }^{18}$ Levels of IgM and IgA against enterobacteria lipopolysaccharides (LPS) were found to be markedly greater in patients with MDD vs those of nondepressed controls. ${ }^{30}$ Current research indicates that IgM and IgA against LPS 
of translocated bacteria serve to amplify immune pathways seen in the pathophysiology of chronic MDD. ${ }^{30,31}$ Further research is indicated to deduce whether bacterial translocation with subsequent immune response induces MDD in susceptible individuals, or whether translocation occurs secondary to the systemic inflammation seen in MDD.

The makeup of the GI microbiome is fundamentally altered in patients with MDD, with a marked reduction in both microorganism diversity and density. ${ }^{30}$ Patients with MDD have been shown to have increased levels of Alistipes, a bacterium that also is elevated in chronic fatigue syndrome and irritable bowel syndrome (IBS), diagnoses that are associated with MDD. ${ }^{32-34}$ Lower counts of Bifidobacterium and Lactobacillus are documented in both MDD and IBS patients as well. ${ }^{35}$ Decreased Bifidobacterium and Lactobacillus might indicate a causal rather than correlative relationship as these bacterium take the precursor monosodium glutamate to create $\gamma$-aminobutyric acid (GABA). ${ }^{36}$

\section{Psychobiotics and Mental Health}

The pathophysiology of the bidirectional communication between the gut and the brain offers an attractive approach for treatment modalities. Currently, the research into probiotic supplementation to treat mental disorders, such as anxiety and depression, is still in its infancy, and treatment guidelines do not support their routine administration. There is great promise in the use of probiotics to ameliorate psychiatric symptomatology, referred to by many in the field as psychobiotics.

One pathophysiology of the stress response seen in anxiety can be traced to the HPA axis and increased cortisol levels, with downstream effects on the microbiome through modification of the migrating motor complexes. Healthy volunteers tasked with taking a trademarked galactooligosaccharide prebiotic daily for 3 weeks had a reduced salivary cortisol awakening response compared with that of a placebo (maltodextrin). The same group demonstrated decreased attentional vigilance to negative information in a dot-probe task compared with attentional vigilance with positive information. ${ }^{37}$ It is possible that this was due to the decreased stress re- sponse secondary to probiotic consumption. In mice models, a probiotic consisting of Lactobacillus helveticus and Bifidobacterium longum (B longum) (bacterium that are decreased in GAD and MDD) demonstrated anxiolytic-like behavior. The same formulation also demonstrated beneficial psychological effects in healthy human volunteers. ${ }^{22}$ In mice models, Lactobacillus feeding was superior to citalopram in anxiolysis and in cognitive functioning. ${ }^{38}$

Like GAD, the pathophysiology of the GBA in MDD is an attractive target for psychobiotic therapy. Although current research is not yet sufficient to create general guidelines or recommendations for the routine administration of psychobiotics, it holds significant promise as an effective primary and/or adjunct treatment. In patients with IBS, administration of $B$ longum reduced depression and increased quality of life. This same study demonstrated that probiotic administration was associated with reduced limbic activity in the brain. ${ }^{39}$ In MDD, the hippocampus demonstrates altered expression of various transcription factors and cellular metabolism. ${ }^{40}$ In a double-blind placebo-controlled trial, Lactobaccillus rhamnosus supplementation in postnatal mothers resulted in less severe depressive symptoms reported.$^{41}$ Furthermore, probiotic supplementation consisting of Lactobacillus acidophilus, Lactobacillus casei, and Bifidobacterium bifidum in patients with MDD for 8 weeks had significant decreases in score on the Beck Depression Inventory scale. ${ }^{42}$ Also, a meta-analysis of probiotic administration on depression scales demonstrated appreciably lower scores after administration in both patients with MDD and healthy patients aged 60 years, although these results were found to be correlative. ${ }^{43}$ However, while promising, another meta-analysis of 10 randomized controlled trials found probiotic supplementation had no significant effect on mood. ${ }^{44}$

\section{THE ROLE OF DIET}

Although there has been tremendous focus on new and improved therapeutics to address MH conditions, such as depression and anxiety, there also has been renewed interest in the fundamental importance and benefit of a wholesome 
diet. Recent literature has shown how diet may play a pivotal role in the development and severity of mental illness and holds promise as another potential target for treatment. A 2010 crosssectional population study of more than 1000 adult women aged 20 to 93 years demonstrated that women with a largely Western dietary pattern (ie, largely composed of processed meats, pizza, chips, hamburgers, white bread, sugar, flavored milk drinks, and beer) were more likely to have dysthymic disorder or major depression, whereas women in this same cohort with a more traditional dietary pattern (ie, composed mainly of vegetables, fruit, lamb, beef, fish, and whole grains) were found to have significantly reduced odds for depression or dysthymic disorder as well as anxiety disorders. ${ }^{45}$

Several other large-scale population studies such as the SUN cohort study, Hordaland Health study, Whitehall II cohort study, and RHEA mother and baby cohort study have demonstrated similar findings: that a more wholesome diet composed mainly of lean meats, vegetables, fruits, and whole grains was associated with significantly reduced risk of depression compared with a largely processed, high fat, and high sugar diet. This trend also has been observed in children and adolescents and is of particular importance when considering that many psychological and psychiatric problems tend to arise in the formative and often turbulent years prior to adulthood. ${ }^{46}$

The causal relationship between diet and MH may be better understood by taking a closer look at a crucial intermediate factor: the gut microbiome. The interplay between diet and intestinal microbiome was well elucidated in a landmark 2010 study by De Filippo and colleagues. ${ }^{47}$ In this study, the microbiota of 14 healthy children from a small village in Burkina Faso (BF) were compared with those of 15 healthy children from an urban area of Florence, Italy (EU). The BF children were reported to consume a traditional rural African diet that is primarily vegetarian, rich in fiber, and low in animal protein and fat, whereas the EU children were noted as consuming a typical Western diet low in fiber but rich in animal protein, fat, sugar, and starch. Comparison revealed that EU children had a higher $\mathrm{F} / \mathrm{B}$ ratio than their BF counterparts, a metric that has been associated with obesity. ${ }^{47}$ Furthermore, increased exposure to environmental microbes associated with a fiber-rich diet has been postulated to increase the richness of intestinal flora and serve as a protective factor against noninfectious and inflammatory colonic diseases, which are found to be more prevalent in Western nations whose diets lack fiber. BF children were found to have increased microbial diversity and increased abundance of bacteria capable of producing SCFA relative to their EU counterparts, both of which have a positive influence on the gut, systemic inflammation, and $\mathrm{MH}^{47}$

\section{CONCLUSIONS}

Diet has a powerful impact on the intestinal microbiome, which in turn directly impacts our physical and $\mathrm{MH}$ in myriad ways. The well-known benefits of a wholesome, nutritious, and well-varied diet include reduced cardiovascular risk, improved glycemic control, GI regularity, and decreased depression. Along with a balanced diet, patients may achieve further benefit with the addition of probiotics.

With regard to psychiatry in particular, increased awareness of the intimate relationship between the gut and the brain is expected to have profound implications for the field. Given this mounting data, immunology, microbiology, and GI pathophysiology should be included in future discussions regarding MH. Their application will likely improve both somatic and mental well-being. We anticipate that newly discovered probiotics and other psychobiotic formulations will be routinely included in a psychiatrist's pharmacopeia in the near future. Unfortunately, as is clear from our review of the current literature, we do not yet have specific interventions targeting the intestinal microbiome to recommend for the management of specific psychiatric conditions. However, this should not deter further exploring diet modification and psychobiotic supplementation as a means of impacting the intestinal microbiome in the pursuit of psychiatric symptom relief.

Dietary modification is already a standard component of sound primary care medicine, designed to mitigate risk for cardiovascular 
disease. This exploration can occur as part of otherwise standard psychiatric care and be used as a form of behavioral activation for the patient. Furthermore, explaining the interconnectedness of the mind, brain, and body along with the rationale for experimentation could further help destigmatize the experience of mental illness.

\section{Author disclosures}

The authors report no actual or potential conflicts of interest with regard to this article.

\section{Disclaimer}

The opinions expressed herein are those of the authors and do not necessarily reflect those of Federal Practitioner, Frontline Medical Communications Inc., the US Government, or any of its agencies. This article may discuss unlabeled or investigational use of certain drugs. Please review the complete prescribing information for specific drugs or drug combinations -including indications, contraindications, warnings, and adverse effects-before administering pharmacologic therapy to patients.

\section{References}

1. Diaz Heijtz R, Wang S, Anuar F, et al. Normal gut microbiota modulates brain development and behavior. Proc Natl Acad Sci USA. 2011;108(7):3047-3052. doi:10.1073/pnas.1010529108

2. Tomkovich S, Jobin C. Microbiota and host immune responses: a love-hate relationship. Immunology. 2016;147(1):1-10. doi:10.1111/imm.12538

3. Bruce-Keller AJ, Salbaum JM, Berthoud HR. Harnessing gut microbes for mental health: getting from here to there. Biol Psychiatry. 2018;83(3):214-223. doi:10.1016/j.biopsych.2017.08.014

4. Patterson E, Cryan JF, Fitzgerald GF, Ross RP, Dinan TG, Stanton C. Gut microbiota, the pharmabiotics they produce and host health. Proc Nutr Soc. 2014;73(4):477-489. doi:10.1017/S0029665114001426

5. Mayer EA, Tillisch K, Gupta A. Gut/brain axis and the microbiota. J Clin Invest. 2015;125(3):926-938. doi:10.1172/JCI76304

6. Lazar V, Ditu LM, Pircalabioru GG, et al. Aspects of gut microbiota and immune system interactions in infectious diseases, immunopathology, and cancer. Front Immunol. 2018;9:1830. doi:10.3389/fimmu.2018.01830

7. Rakoff-Nahoum S, Paglino J, Eslami-Varzaneh F, Edberg S, Medzhitov R. Recognition of commensal microflora by tolllike receptors is required for intestinal homeostasis. Cell. 2004;118(2):229-241. doi:10.1016/j.cell.2004.07.002

8. Ghosh S, van Heel D, Playford RJ. Probiotics in inflammatory bowel disease: is it all gut flora modulation? Gut. 2004;53(5):620-622. doi:10.1136/gut.2003.034249

9. Fedorak RN. Probiotics in the management of ulcerative colitis. Gastroenterol Hepatol (NY). 2010;6(11):688-690.

10. Ianiro $\mathrm{G}$, Mullish $\mathrm{BH}$, Kelly $\mathrm{CR}$, et al. Screening of faecal microbiota transplant donors during the COVID-19 outbreak: suggestions for urgent updates from an international expert panel. Lancet Gastroenterol Hepatol. 2020;5(5):430-432. doi:10.1016/S2468-1253(20)30082-0

11. Verna EC, Lucak S. Use of probiotics in gastrointestinal disorders: what to recommend? Therap Adv Gastroenterol. 2010;3(5):307-319. doi:10.1177/1756283X10373814

12. Hegazy SK, El-Bedewy MM. Effect of probiotics on proinflammatory cytokines and NF-kappaB activation in ulcerative colitis. World J Gastroenterol. 2010;16(33):4145-4151. doi:10.3748/wjg.v16.i33.4145

13. Kleiman SC, Watson HJ, Bulik-Sullivan EC, et al. The intestinal microbiota in acute anorexia nervosa and during renourishment: relationship to depression, anxiety, and eating disorder psychopathology. Psychosom Med. 2015;77(9):969-981. doi:10.1097/PSY.0000000000000247

14. Rodes L, Paul A, Coussa-Charley M, et al. Transit time affects the community stability of Lactobacillus and Bifidobacterium species in an in vitro model of human colonic microbiotia. Artif Cells Blood Substit Immobil Biotechnol. 2011;39(6):351-356. doi:10.3109/10731199.2011.622280

15. Jiang $\mathrm{HY}$, Zhang $\mathrm{X}, \mathrm{Yu} \mathrm{ZH}$, et al. Altered gut microbiota profile in patients with generalized anxiety disorder. J Psychiatr Res. 2018;104:130-136. doi:10.1016/j.jpsychires.2018.07.007

16. van de Wouw M, Boehme M, Lyte JM, et al. Shortchain fatty acids: microbial metabolites that alleviate stress-induced brain-gut axis alterations. J Physiol. 2018;596(20):4923-4944 doi:10.1113/JP276431.

17. Morris G, Berk M, Carvalho A, et al. The role of the microbial metabolites including tryptophan catabolites and short chain fatty acids in the pathophysiology of immuneinflammatory and neuroimmune disease. Mol Neurobiol. 2017;54(6):4432-4451 doi:10.1007/s12035-016-0004-2.

18. Kelly JR, Kennedy PJ, Cryan JF, Dinan TG, Clarke G, Hyland NP. Breaking down the barriers: the gut microbiome, intestinal permeability and stress-related psychiatric disorders. Front Cell Neurosci. 2015;9:392. doi:10.3389/fncel.2015.00392

19. Duivis HE, Vogelzangs N, Kupper N, de Jonge P, Penninx BW. Differential association of somatic and cognitive symptoms of depression and anxiety with inflammation: findings from the Netherlands Study of Depression and Anxiety (NESDA). Psychoneuroendocrinology. 2013;38(9):1573-1585. doi:10.1016/j.psyneuen.2013.01.002

20. Miller $\mathrm{AH}$, Raison $\mathrm{CL}$. The role of inflammation in depression: from evolutionary imperative to modern treatment target. Nat Rev Immunol. 2016;16(1):22-34. doi:10.1038/nri.2015.5

21. Morilak DA, Frazer A. Antidepressants and brain monoaminergic systems: a dimensional approach to understanding their behavioural effects in depression and anxiety disorders. Int J Neuropsychopharmacol. 2004;7(2):193-218. doi:10.1017/S1461145704004080

22. Messaoudi M, Lalonde R, Violle N, et al. Assessment of psychotropic-like properties of a probiotic formulation (Lactobacillus helveticus R0052 and Bifidobacterium longum R0175) in rats and human subjects. Br J Nutr. 2011;105(5):755-764. doi:10.1017/S0007114510004319

23. Ishak WW, Mirocha J, James D. Quality of life in major depressive disorder before/after multiple steps of treatment and one-year follow-up. Acta Psychiatr Scand. 2014;131(1):51-60. doi:10.1111/acps.12301

24. El Aidy S, Dinan TG, Cryan JF. Immune modulation of the brain-gut-microbe axis. Front Microbiol. 2014;5:146. doi:10.3389/fmicb.2014.00146

25. Browning KN, Verheijden S, Boeckxstaens GE. The vagus nerve in appetite regulation, mood, and intestinal inflammation. Gastroenterology. 2017;152(4):730-744. doi:10.1053/j.gastro.2016.10.046

26. Berthoud HR, Neuhuber WL. Functional and chemical anatomy of the afferent vagal system. Auton Neurosci. 2000;85(1-3):1-7. doi:10.1016/S1566-0702(00)00215-0

27. Nahas Z, Marangell LB, Husain MM, et al. Two-year outcome of vagus nerve stimulation (VNS) for treatment of major depressive episodes. J Clin Psychiatry. 2005;66(9). doi:10.4088/jcp.v66n0902

28. Forsythe P, Bienenstock J, Kunze WA. Vagal pathways for microbiome-brain-gut axis communication. In: Microbial Endocrinology: The Microbiota-Gut-Brain Axis in Health and Disease. New York, NY: Springer; 2014:115-133.

29. Miller $\mathrm{AH}$, Raison $\mathrm{CL}$. The role of inflammation in depression: from evolutionary imperative to modern treatment target. Nat Rev Immunol. 2015;16(1):22-34. doi:10.1038/nri.2015.5

30. Mass M, Kubera M, Leunis JC. The gut-brain barrier in major depression: intestinal mucosal dysfunction with an increased translocation of LPS from gram negative enterobacteria (leaky gut) plays a role in the inflammatory 
pathophysiology of depression. Neuro Endocrinol Lett. 2008;29(1):117-124.

31. Goehler LE, Gaykema RP, Opitz N, Reddaway R, Badr N, Lyte M. Activation in vagal afferents and central autonomic pathways: early responses to intestinal infection with Campylobacter jejuni. Brain, Behav Immun. 2005;19(4):334-344. doi:10.1016/j.bbi.2004.09.002

32. Stevens BR, Goel R, Seungbum K, et al. Increased human intestinal barrier permeability plasma biomarkers zonulin and FABP2 correlated with plasma LPS and altered gut microbiome in anxiety or depression. Gut. 2018;67(8):15551557. doi:10.1136/gutjnl-2017-314759

33. Kelly JR, Borre Y, O'Brien C, et al. Transferring the blues: depression-associated gut microbiota induces neurobehavioural changes in the rat. J Psychiatr Res. 2016;82:109118. doi:10.1016/j.jpsychires.2016.07.019

34. Jiang $\mathrm{H}$, Ling $\mathrm{Z}$, Zhang $\mathrm{Y}$, et al. Altered fecal microbiota composition in patients with major depressive disorder. Brain Behav Immun. 2015;48:186-194. doi:10.1016/.jbbi.2015.03.016

35. Frémont M, Coomans D, Massart S, De Meirleir K. Highthroughput 16S rRNA gene sequencing reveals alterations of intestinal microbiota in myalgic encephalomyelitis/ chronic fatigue syndrome patients. Anaerobe. 2013;22:5056. doi:10.1016/j.anaerobe.2013.06.002

36. Saulnier DM, Riehle K, Mistretta TA, et al. Gastrointestinal microbiome signatures of pediatric patients with irritable bowel syndrome. Gastroenterol. 2011;141(5):1782-1791. doi:10.1053/j.gastro.2011.06.072

37. Schmidt K, Cowen PJ, Harmer CJ, Tzortzis G, Errington $S$, Burnet PW. Prebiotic intake reduces the waking cortisol response and alters emotional bias in healthy volunteers. Psychopharmacology (Berl). 2015;232(10):1793-1801. doi:10.1007/s00213-014-3810-0

38. Liang S, Wang T, Hu X, et al. Administration of Lactobacillus helveticus NS8 improves behavioral, cognitive, and biochemical aberrations caused by chronic restraint stress. Neuroscience. 2015;310:561-577. doi:10.1016/j.neuroscience

39. Pinto-Sanchez MI, Hall GB, Ghajar K, et al. Probiotic Bifi- dobacterium longum NCC3001 reduces depression scores and alters brain activity: a pilot study in patients with irritable bowel syndrome. Gastroenterology. 2017;153(2):448459. doi:10.1053/j.gastro.2017.05.003

40. Sequeira A, Klempan T, Canetti L, Benkelfat C, Rouleau GA, Turecki G. Patterns of gene expression in the limbic system of suicides with and without major depression. Mol Psychiatry. 2007;12(7):640-555. doi:10.1038/sj.mp.4001969

41. Slykerman RF, Hood F, Wickens K, et al. Effect of Lactobacillus rhamnosus HNOO1 in pregnancy on postpartum symptoms of depression and anxiety: a randomised double-blind placebo-controlled trial. EBioMedicine. 2017;24:159-165. doi:10.1016/j.ebiom.2017.09.013

42. Akkasheh G, Kashani-Poor Z, Tajabadi-Ebrahimi M, et al. Clinical and metabolic response to probiotic administration in patients with major depressive disorder: a randomized, double-blind, placebo-controlled trial. Nutrition. 2016;32(3):315-320. doi:10.1016/j.nut.2015.09.003

43. Huang R, Wang K, Hu J. Effect of probiotics on depression: a systematic review and meta-analysis of randomized controlled trials. Nutrients. 2016;8(8):483. doi:10.3390/nu8080483

44. Ng QX, Peters C, Ho CY, Lim DY, Yeo WS. A metaanalysis of the use of probiotics to alleviate depressive symptoms. J Affect Disord. 2018;228:13-19. doi:10.1016/j.jad.2017.11.063

45. Jacka FN, Pasco JA, Mykletun A, et al. Association of Western and traditional diets with depression and anxiety in women. Am J Psychiatry. 2010;167(3):305-311. doi:10.1176/appi.ajp.2009.09060881.

46. Jacka FN, Mykletun A, Berk M. Moving towards a population health approach to the primary prevention of common mental disorders. BMC Med. 2012;10:149. doi: 10.1186/1741-7015-10-149

47. De Filippo C, Cavalieri D, Di Paola Met, et al. Impact of diet in shaping gut microbiota revealed by a comparative study in children from Europe and rural Africa. Proc Natl Acad Sci U S A. 2010;107(33):14691-14696. doi:10.1073/pnas.1005963107 